\title{
EXISTENCE AND RELAXATION RESULTS FOR NONLINEAR EVOLUTION INCLUSIONS REVISITED
}

\author{
S. MIGÓRSKI \\ Jagellonian University \\ Institute for Information Sciences \\ ul. Nawojki 11, 30072 Cracow Poland
}

(Received August, 1994; Revised February, 1995)

\begin{abstract}
In this paper we confirm the validity of some recent results of $\mathrm{Hu}$, Lakshmikantham, Papageorgiou [4] and Papageorgiou [13] concerning the existence and relaxation for nonlinear evolution inclusions. We fill a gap in the proofs of these results due to the use of incorrect Nagy's compactness embedding theorem.
\end{abstract}

Key words: Evolution Triple, Compact Embedding, Monotone and Hemicontinuous Map, Evolution Inclusion, Orientor Field, Relaxation.

AMS (MOS) subject classifications: 34G20.

\section{Introduction}

Recently $\mathrm{Hu}$, Lakshmikantham and Papageorgiou [4] studied the properties of the solution set of nonlinear evolution inclusion driven by a time dependent maximal monotone coercive operators defined on an evolution triple $\left(V, H, V^{\prime}\right)$. However, the main existence theorem of their paper (Theorem 3.1 in [4]) as well as the relaxation theorem (Theorem 3.2 in [4] ) have a gap in their proofs and therefore, in our opinion, these proofs are incorrect. The same remark concerns the proof of another result on existence of solutions to differential inclusion due to Papageorgiou (see Theorem 3.1 of [13]). Namely, in [4] and [13] the authors have exploited several times the result of Nagy (see Theorem 2 in [7]), which says that $\mathcal{W}$ (the space where the solution of the inclusion is sought) is compactly embedded into $C(0, T ; H)$ (the space of continuous functions from the time interval $[0, T]$ into $H$ ); see the notation in Section 2 below. Very recently, the author of this paper gave an example (see [6]) which shows that the above result of Nagy is false. For this reason, the proofs of existence and relaxation theorems cannot follow directly from arguments presented in [4] and [13].

The purpose of this paper is to fill the gap due to the use of Nagy's incorrect result and to establish in this way the validity of some earlier results of [4] and [13].

As the referee noticed, it is important to observe that the proofs of Theorems 3.1 and 3.2 in [4] and Theorem 3.1 in [13] remain valid (even without assuming $V$ to be a separable Hilbert space) provided $x_{0} \in V$, i.e., when the initial condition is more regular. In this situation, the result of Nagy [7] can be omitted. Indeed, in this case the solution set of the evolution inclusion is sequentially compact in $C(0, T ; w-V)$ (see [1] or [10]). Hence Corollary 4, p. 85 of Simon [15] guarantees the compactness of this set in $C(0, T ; H)$.

In the light of the above observation, the present paper shows that it is not necessary to 
restrict $x_{0}$ in $V$, that is, we will prove that even if the initial datum is nonsmooth the solution set remains compact in $C(0, T ; H)$. We note that the new mathematical argument of this paper is based on the fact that the solution map (which assigns to the right-hand side the solution) of the associated evolution equation is sequentially continuous from ' $\mathcal{G}^{\prime}$ ' endowed with its weak topology into $C(0, T ; H)$ (see Proposition 3.1). Nonetheless, for the sake of completeness and clarity, we will give (following [4] and [13]) the main steps of the proofs of two existence theorems and a relaxation theorem established in [4] and [13] indicating places where the mentioned above result of Nagy has to be replaced by Proposition 3.1. Note that Theorem 3.1 has been already established for $p=q=2$ in [12], while Theorem 3.2 can be found in a more general setting in [14]. Another relevant work is [11], where a controlled evolution inclusion with control constraints was examined.

\section{Preliminaries}

Let $H$ be a separable Hilbert space and let $V$ be a subspace of $H$ carrying the structure of a separable reflexive Banach space, which embeds into $H$ densely and continuously. Identifying $H$ with its dual, we have the Gelfand triple (see e.g., $[2,5,16]) V \subset H \subset V^{\prime}$, where all embeddings are continuous and dense. Moreover, we assume in this paper that these embeddings are also compact. We denote by $\langle\cdot, \cdot\rangle$ the duality of $V$ and its dual $V^{\prime}$ as well as the inner product on $H$, by $\|\cdot\|,|\cdot|$ and $\|\cdot\|_{V^{\prime}}$ the norms in $V, H$ and $V^{\prime}$, respectively. Given a fixed real number $T>0$ and $2 \leq p<+\infty$, we introduce the following spaces $\mathscr{V}=L^{p}(0, T ; V), \mathcal{H} 6=L^{p}(0, T ; H)$, $\mathcal{H}^{\prime}=L^{q}(0, T ; H), \mathcal{q}^{\prime}=L^{q}\left(0, T ; V^{\prime}\right),(1 / p+1 / q=1)$ and $\mathscr{W}=\left\{w \in \mathcal{V} \mid w^{\prime} \in \mathcal{q}^{\prime}\right\}$, where the derivative is understood in the sense of vector valued distributions. Clearly $W \subset \mathscr{V} \subset \mathcal{H} \subset \mathcal{V}^{\prime}$. The pairing of $\mathscr{V}$ and $\mathscr{V}^{\prime}$ and the duality between $\mathfrak{H}$ and $\mathcal{G}^{\prime}$ are denoted by $\langle\langle f, v\rangle\rangle=\int_{0}^{T}\langle f(s), v(s)\rangle d s$. Given a Banach space $\mathscr{S}$, the symbols $w-\mathscr{S}, s-\mathscr{S}$ are always used to indicate the space $\mathscr{6}$ equipped with the weak and the strong (norm) topology, respectively.

Let $(\Omega, \Sigma, \mu)$ be a measure space, $X$ be a separable Banach space. By $\mathscr{P}_{f(c)}(X)$ and $\mathscr{P}_{(w) k(c)}(X)$ we denote respectively the family of all nonempty, closed, (convex) subsets of $X$ and the family of all nonempty, (weak-) compact, (convex) subsets of $X$. A multifunction $F$ defined on $\Omega$ with values in the space $2^{X}$ of all nonempty subsets of $X$ is called measurable if $F^{-}(E):=$ $\{\omega \in \Omega: F(\omega) \cap E \neq \emptyset\} \in \Sigma$, for every closed set $E \subset X$. F is called graph measurable if $G r F:=$ $\{(\omega, x) \in \Omega \times X: x \in F(\omega)\} \in \Sigma \times \mathfrak{B}(X)$ (here $\mathfrak{B}(X)$ is the family of all Borel subsets of $X$ ). We denote by $S_{F}^{p}(1 \leq p \leq \infty)$ the set of all selectors of $F$ that belong to $L^{p}(\Omega ; X)$ i.e., $S_{F}^{p}=\{f \in$ $L^{p}(\Omega ; X): f(\omega) \in F(\omega) \mu$ a.e. $\}$. We know that $S_{F}^{p} \neq \emptyset$ if and only if $\omega \mapsto \inf \{\|x\|: x \in F(\omega)\} \in$ $L_{+}^{p}$. The set $S_{F}^{p}$ is said to be decomposable (see e.g., [9]) if $A \in \mathfrak{B}(\Omega)$ (the Borel $\sigma$-field of $\Omega$ ) and $f_{1}, f_{2} \in S_{F}^{p}$ imply $\chi_{A} f_{1}+\left(1-\chi_{A}\right) f_{2} \in S_{F}^{p}$. Let $\left(Y, \tau_{Y}\right),\left(Z, \tau_{Z}\right)$ be Hausdorff topological spaces. A multifunction $G: Y \rightarrow 2^{Z}$ is said to be $\left(\tau_{Y}-\tau_{Z}\right)$ upper semicontinuous (u.s.c.) (respectively, lower semicontinuous (l.s.c.)), if for every $C \subseteq Z$ closed in $\tau_{Z}$ topology, $G^{-}(C)$ (respectively, $\left.G^{+}(C):=\{y \in Y: G(y) \subseteq C\}\right)$ is closed in $\tau_{Y}$ topology in $Y$.

\section{Existence and Relaxation Theorems}

In this section we examine the continuity properties of the solution map of the Cauchy problem for the evolution equation associated with the following nonlinear inclusion:

$$
\left\{\begin{array}{l}
\dot{x}(t)+A(t, x(t)) \in F(t, x(t)) \text { a.e. } t \in(0, T), \\
x(0)=x_{0}
\end{array}\right.
$$


Then we will present concisely the proofs of existence and relaxation results by $\mathrm{Hu}$, Lakshmikantham and Papageorgiou [4] and Papageorgiou [13].

We introduce the following.

Definition 3.1: A function $x \in \mathscr{W}$ is called a solution of (3.1) if and only if

$$
\left\{\begin{array}{l}
\dot{x}(t)+A(t, x(t))=f(t) \text { a.e. } t \in(0, T) \text { in } V^{\prime} \\
x(0)=x_{0}
\end{array}\right.
$$

with $f \in S_{F(\cdot, x(\cdot))}^{q}$.

The following hypotheses will be used in the sequel.

$\underline{H(A):} \quad A:[0, T] \times V \rightarrow V^{\prime}$ is an operator such that

(1) $\quad t \rightarrow A(t, v)$ is measurable from $[0, T]$ to $V^{\prime}$,

(2) $v \rightarrow A(t, v)$ is monotone and hemicontinuous from $V$ to $V^{\prime}$,

(3) $\|A(t, v)\|_{V^{\prime}} \leq a(t)+b\|v\|^{p-1}$, a.e. $t \in(0, T), \forall v \in V$ with $a \in L_{+}^{q}(0, T), b>0$,

(4) $\langle A(t, v), v\rangle \geq c\|v\|^{p}$, a.e. $t \in(0, T)$ with $c>0$.

$\underline{H(F)_{1}}: \quad F:[0, T] \times H \rightarrow \mathcal{P}_{f c}(H)$ is a multifunction such that

(1) $\quad(t, x) \rightarrow F(t, x)$ is graph measurable,

(2) $\quad G r F(t, \cdot)$ is sequentially closed in $H \times(w-H)$, a.e. $t \in(0, T)$,

(3) $|F(t, x)| \leq a_{1}(t)+b_{1}(t)|x|^{2 / q}$, a.e. $t \in(0, T)$ with $a_{1}, b_{1} \in L_{+}^{q}(0, T)$.

$\underline{H(F)_{2}}: \quad F:[0, T] \times H \rightarrow \Phi_{f}(H)$ is a multifunction such that

(1) $\quad H(F)_{1}(1)$ holds,

(2) $\quad x \mapsto F(t, x)$ is l.s.c.,

(3) $\quad H(F)_{1}(3)$ holds.

The hypotheses $H(A), H(F)_{1}, H(F)_{2}$ for $p=q=2$ coincide with the ones of [13] and [4].

It is well-known (see Theorem 4.2, p. 167 of Barbu [2] or Theorem 1.2, p. 162 of Lions [5]) that if $H(A)$ holds, $f \in \mathcal{F}^{\prime}$ and $x_{0} \in H$, then evolution equation (3.2) admits a unique solution in $\mathscr{W}$. We consider below the solution map $r: \mathfrak{J G}^{\prime} \rightarrow \mathcal{W}$ for $(3.2)$ defined by $r(f)=x$, where $x$ denotes the solution to $(3.2)$.

Proposition 3.1: If hypothesis $H(A)$ holds and $x_{0} \in H$, then the solution map for (3.2) is continuous from $w-\mathcal{J G}^{\prime}$ into $w-\mathcal{W}$ and from $w-\mathcal{J G}^{\prime}$ into $C(0, T ; H)$.

Proof: Let $f_{n}, f \in \mathcal{H}^{\prime}$ be such that

$$
f_{n} \rightarrow f \text { in } w-\mathfrak{G}^{\prime}
$$

and let $x_{n}=r\left(f_{n}\right) \in \mathcal{W}$ be the sequence of unique solutions to (3.2) corresponding to $f_{n}$. From the classical a priori estimates of the solutions to parabolic equation (see Chapter 2.1 of Lions $[5]$ ), it follows that $\left\{x_{n}\right\}$ remains in a bounded subset of $\mathcal{W}$. By extracting a subsequence, if necessary, we may assume that

$$
x_{n} \rightarrow x \text { in } w-W
$$

for some $x \in \mathscr{W}$. Since $\mathscr{W} \subset \mathcal{H}$ compactly (see Theorem 5.1 of Lions [5], p. 58), we have also

$$
x_{n} \rightarrow x \text { in } s-\mathfrak{H} \text {. }
$$


Consider the convex set $\mathscr{W}_{x_{0}}=\left\{w \in W: w(0)=x_{0}\right\}$. This set is closed and hence weakly closed in $\mathscr{W}$, in virtue of the fact that $\mathscr{W} \subset C(0, T ; H)$ continuously (see Proposition 23.23, p. 422 of Zeidler $[16])$. Since $x_{n} \in \mathcal{W}_{x_{0}}$, from (3.4) we deduce that

$$
x(0)=x_{0}
$$

We claim that

$$
\limsup _{n}\left\langle\left\langle\widehat{A}\left(x_{n}\right), x_{n}-x\right\rangle\right\rangle \leq 0,
$$

where by $\widehat{A}: q \rightarrow q^{\prime}$ we have denoted the Nemitsky operator corresponding to $A$, i.e., $(\widehat{A} v)(t)=$ $A(t, v(t))$ for a.e. $t$. Multiplying the equation $\dot{x}_{n}+\widehat{A}\left(x_{n}\right)=f_{n}$ in duality by $x_{n}-x$, using the integration by parts formula (see e.g., [1], [16]) and (3.6), we obtain

$$
\left\langle\left\langle\dot{x}, x_{n}-x\right\rangle\right\rangle+\frac{1}{2}\left|x_{n}(T)-x(T)\right|^{2}+\left\langle\left\langle\widehat{A}\left(x_{n}\right), x_{n}-x\right\rangle\right\rangle=\left\langle\left\langle f_{n}, x_{n}-x\right\rangle\right\rangle .
$$

From (3.3), (3.4) and (3.5), by taking the limit in (3.8) and dropping the positive term, we get

$$
\limsup _{n}\left\langle\left\langle\widehat{A}\left(x_{n}\right), x_{n}-x\right\rangle\right\rangle \leq \lim _{n}\left(\left\langle\left\langle f_{n}, x_{n}-x\right\rangle\right\rangle-\left\langle\left\langle\dot{x}, x_{n}-x\right\rangle\right\rangle\right)=0 .
$$

This proves the claim.

We are going to show now that by extracting further subsequences, we may assume that $x_{n} \rightarrow x$ in $C(0, T ; H)$. We set $\varrho_{n}(t)=\left\langle A\left(t, x_{n}(t)\right)-A(t, x(t)), x_{n}(t)-x(t)\right\rangle$ for every $n$ and a.e. $t \in$ $(0, T)$. Applying Fatou's Lemma to functions $\varrho_{n}$ (by $H(A)(2)$ we know that $\varrho_{n}$ are nonnegative), and using (3.4) and (3.7), we have that

$$
\begin{gathered}
0 \leq \int_{0}^{T} \liminf \varrho_{n}(s) d s \leq \lim _{n} \inf \int_{0}^{T} \varrho_{n}(s) d s \leq \limsup _{n} \int_{0}^{T} \varrho_{n}(s) d s \\
\leq \limsup _{n} \int_{0}^{T}\left\langle A\left(s, x_{n}(s)\right), x_{n}(s)-x(s)\right\rangle d s-\lim _{n} \int_{0}^{T}\left\langle A(s, x(s)), x_{n}(s)-x(s)\right\rangle d s \\
=\limsup _{n}\left\langle\left\langle\widehat{A}\left(x_{n}\right), x_{n}-x\right\rangle\right\rangle \leq 0 .
\end{gathered}
$$

From these inequalities, we deduce that $\lim _{n} \int_{0}^{T} \varrho_{n}(s) d s=0$, which clearly implies that $\varrho_{n} \rightarrow 0$ strong ly in $L^{1}(0, T)$. Therefore, we may assume, by taking a next subsequence, that

$$
\varrho_{n}(t) \rightarrow 0 \text { a.e. } t \in(0, T) .
$$

Using hypothesis $H(A)(3)(4)$, for a.e. $t \in(0, T)$, we get

$$
\begin{gathered}
\varrho_{n}(t) \geq c\left\|x_{n}(t)\right\|^{p}-b\|x(t)\|\left\|x_{n}(t)\right\|^{p-1} \\
-\left\|x_{n}(t)\right\|\left(a(t)+b\|x(t)\|^{p-1}\right)-a(t)\|x(t)\|+c\|x(t)\|^{p} .
\end{gathered}
$$

From the above inequality and (3.9), it follows that $\left\{\left\|x_{n}(t)\right\|\right\}$ is bounded for a.e. $t \in(0, T)$ and $n \geq n_{0}$. Thus we have shown that the sequence $\left\{x_{n}\right\}$ belongs to a bounded set of $L^{\infty}(0, T ; V)$. Moreover, since $\left\{\dot{x}_{n}\right\}$ lies in a bounded subset of $q^{\prime}$ and $V \subset H$ compactly, we deduce by a version of the Arzelá-Ascoli theorem (compare Corollary 4, §8 of Simon [15]) that $x_{n} \rightarrow x$ in 
$C(0, T ; H)$.

As in the proof of Theorem 3.1 in [13], we show that $x=r(f)$ is a solution to (3.2). From the uniqueness of solutions to (3.2), we infer that the whole sequence $\left\{x_{n}\right\}$ converges to $x$ in both $w-\mathcal{W}$ and $C(0, T ; H)$. This completes the proof of the proposition.

Theorem 3.1: (The convex case) If hypotheses $H(A), H(F)_{1}$ hold and $x_{0} \in H$, then (3.1) admits a solution.

Proof:

Step 1. Every solution $x \in \mathscr{W}$ to (3.1) satisfies the following a priori estimates:

$$
\begin{gathered}
|x(t)| \leq M_{1}, \quad \forall t \in[0, T], \\
\|x\|_{W} \leq M_{2},
\end{gathered}
$$

where $M_{i}>0$ for $i=1,2$.

Step 2. The multifunction $\widehat{F}:[0, T] \times H \rightarrow 2^{H}$ given by

$$
\widehat{F}(t, x)=\left\{\begin{array}{cl}
F(t, x) & \text { if }|x| \leq M_{1} \\
F\left(t, \frac{M_{1} x}{|x|}\right) & \text { if }|x|>M_{1}
\end{array}\right.
$$

is $\mathscr{P}_{f c}(H)$-valued. It satisfies $H(F)_{1}(1)(2)$ and $|\widehat{F}(t, x)| \leq \tilde{a}(t)$, a.e. $t$, where $\tilde{a} \in L_{+}^{q}(0, T)$.

Step 3 . The multifunction $\mathscr{B}: \mathscr{Z} \rightarrow 2^{L^{1}(0, T ; H)}$ defined by

$$
\mathscr{R}(f)=S_{\widehat{F}(\cdot, r(f)(\cdot))}^{1},
$$

where $\mathscr{Z}=\left\{f \in \mathcal{J}^{\prime}:|f(t)| \leq \tilde{a}(t)\right.$ a.e. $\left.t\right\}$, is $\mathscr{T}_{f c}(\mathscr{Z})$-valued. By Theorem 4.2 of Papageorgiou [8] and Proposition $3.1, \mathscr{R}_{\mathbb{B}}$ is $\left(\mathrm{w}-\mathfrak{J G}^{\prime}\right) \times\left(\mathrm{w}^{\prime} \mathfrak{G}^{\prime}\right)$ u.s.c. on $\mathscr{Z}$.

Step 4. One applies the Kakutani-Fan fixed point theorem to the multifunction $\mathscr{B}$ finding $f^{*} \in \mathcal{Z}$ such that $f^{*} \in \mathscr{B}\left(f^{*}\right)$. Then $x^{*}:=r\left(f^{*}\right)$ solves (3.1) with $\widehat{F}$ in place of $F$. The same estimate as in Step 1 and (3.12) implies $\widehat{F}\left(t, x^{*}(t)\right)=F\left(t, x^{*}(t)\right)$ for a.e. $t$, which means that $x^{*}$ is a solution to (3.1). This completes the proof of the theorem.

From Theorem 3.1 and (3.11), we obtain the following.

Corollary 3.1: If hypotheses of Theorem 3.1 hold, then the solution set of (3.1) is a nonempty, weakly compact subset of $\mathcal{W}$ and a compact subset of $C(0, T ; H)$.

Theorem 3.2: (The nonconvex case) If hypotheses $H(A), H(F)_{2}$ hold and $x_{0} \in H$, then (3.1) admits a solution.

\section{Proof:}

Step 1. As in the proof of Theorem 3.1, we get for the solutions a priori bounds (3.10) and

Step 2. One verifies that the multifunction $\widehat{F}$ defined in (3.12) is graph measurable, $|\widehat{F}(t, x)| \leq \tilde{a}(t)$, a.e. $t$ and $\widehat{F}(t, \cdot)$ is l.s.c. from $s-H$ into $\mathscr{\Phi}_{f}(H)$.

Step 3. By using Theorem 4.1 of Papageorgiou [8] and Proposition 3.1, one obtains that the multifunction $\mathscr{R}: \mathscr{T} \rightarrow \mathscr{P}_{w k}\left(L^{1}(0, T ; H)\right)$ defined by $(3.13)$ is l.s.c. from $\mathscr{Z}$ (endowed with $w-\mathfrak{H}^{\prime}$ topology) into $s-L^{1}(0, T ; H)$. Moreover, the images of $\mathscr{R}_{0}(\cdot)$ are decomposable sets (see [9]). 
Step 4. Applying the selection theorem of Fryszkowski [3], we get a continuous map $\eta: \mathscr{Z} \rightarrow \mathscr{Z}$ such that $\eta(f) \in \mathscr{B}(f)$ for all $f \in \mathscr{Z}$. Then, by the Schauder-Tikhonov fixed point theorem, one finds $f^{*} \in \mathscr{Z}$ such that $f^{*}=\eta\left(f^{*}\right)$. As in the proof of Theorem 3.1, it is easy to check that $x^{*}=r\left(f^{*}\right)$ solves (3.1) This completes the proof.

Finally, we consider the following "convexified" version fo the Cauchy problem (3.1):

$$
\left\{\begin{array}{l}
\dot{x}(t)+A(t, x(t)) \in \overline{c o} F(t, x(t)) \text { a.e. } t \in(0, T) \\
x(0)=x_{0} .
\end{array}\right.
$$

Denote by $S\left(x_{0}\right)$ the solution set of (3.1) and by $S_{c}\left(x_{0}\right)$ the solution set of (3.14). The relaxation theorem stated below shows that $S\left(x_{0}\right)$ is dense in $S_{c}\left(x_{0}\right)$ for the $C(0, T ; H)$ topology. As regards the orientor field, we need now a hypothesis which is stronger than the ones considered previously.

$\underline{H(F)_{3}}: \quad F:[0, T] \times H \rightarrow \mathscr{\Phi}_{f}(H)$ is a multifunction such that

(1) $\quad F(\cdot, x)$ is measurable on $[0, T]$, for all $x \in H$,

(2) $h(F(t, x), F(t, y)) \leq \vartheta(t)|x-y|$, a.e. $t$ with $\vartheta \in L_{+}^{1}(0, T)$,

(3) $\quad H(F)_{1}(3)$ holds,

where $h(\cdot, \cdot)$ denotes the Hausdorff metric.

Theorem 3.3: Under hypotheses $H(A), H(F)_{3}$ and $x_{0} \in H$, we have $\overline{S\left(x_{0}\right)}=S_{c}\left(x_{0}\right)$, where the closure is taken in the $C(0, T ; H)$ norm topology.

\section{Proof:} converse.

Step 1. From Corollary 3.1, it follows that $\overline{S\left(x_{0}\right)} \subset S_{c}\left(x_{0}\right)$. It is sufficient to prove the

Step 2. Let $\epsilon>0$ and $x \in S_{c}\left(x_{0}\right)$. So (3.2) holds with $f \in S \frac{q}{c o} F(\cdot, x(\cdot))$. As in the proof of Theorem 3.1, one defines the set $\mathscr{Z}$ and gets $|f(t)| \leq \tilde{a}(t)$ a.e.. Due to Theorem 4.1 of Papageorgiou [10] and Proposition 3.1, it is possible to find $f_{1} \in S_{F(\cdot, x(\cdot))}^{1}$ and a symmetric weak neighborhood $U$ of the origin in 'JG' such that $f-f_{1} \in U \cap \mathcal{Z}$ implies $\left\|x-z_{1}\right\|_{C(0, T ; H)} \leq \epsilon$, where $z_{1}=r\left(f_{1}\right)$.

Step 3. By virtue of the Aumann selection theorem, we construct by induction a sequence $\left\{f_{n}\right\} \subseteq \mathcal{J}^{\prime}$ such that

$$
\begin{gathered}
f_{i+1}(t) \in F\left(t, z_{i}(t)\right) \text { a.e. } t \\
\left|f_{i+1}(t)-f_{i}(t)\right| \leq \frac{\epsilon \vartheta(t)}{(i-1) !}\left(\int_{0}^{t} \vartheta(s) d s\right)^{i-1} \text { a.e. } t \\
z_{i}=r\left(f_{i}\right)\left(\text { here } z_{0}=x \in S_{c}\left(x_{0}\right)\right)
\end{gathered}
$$

for every $i \geq 1$.

Step 4. From Step 3, again by Proposition 3.1, we deduce that $z_{n}=r\left(f_{n}\right) \rightarrow r(\widehat{f})=: \widehat{z}$ in $C(0, T ; H)$ and next that $\widehat{z} \in S\left(x_{0}\right)$. Furthermore, due to the inequalities

$$
\left|z_{i+1}(t)-z_{i}(t)\right| \leq \frac{\epsilon}{i !}\left(\int_{0}^{t} \vartheta(s) d s\right)^{i}, \forall i \geq 1
$$


we obtain $\|x-\widehat{z}\|_{C(0, T ; H)} \leq \epsilon \exp \left(\|\vartheta\|_{L^{1}}\right)$. Since $\epsilon>0$ is arbitrary we conclude that $x \epsilon$ $\overline{S\left(x_{0}\right)}$.

\section{Acknowledgements}

The author wishes to thank the referee for his/her remarks and for bringing some additional references to the author's attention.

\section{References}

[1] Ahmed, N.U. and Teo, K.L., Optimal Control of Distributed Parameter Systems, North Holland, New York 1981.

[2] Barbu, V., Nonlinear Semigroups and Differential Equations in Banach Spaces, Noordhoff International Publishing, Leyden, The Netherlands 1976.

[3] Fryszkowski, A., Continuous selections for nonconvex multivalued maps, Studia Math. 76 (1983), 163-174.

[4] Hu, S., Lakshmikantham, V. and Papageorgiou, N.S., On the solution set of nonlinear evolution inclusions, Dynamic Systems and Appl. 1 (1992), 71-82.

[5] Lions, J.L., Quelques Méthodes de Résolution des Problémes aux Limites non Linéaires, Dunod, Paris 1969.

[6] Migórski, S., A counterexample to a compact embedding theorem for functions with values in a Hilbert space, Proc. Amer. Math. Society (1995), preprint CSJU-04/94.

[7] Nagy, E.V., A theorem on compact embedding for functions with values in an infinite dimensional Hilbert space, Ann. Univ. Sci. Budapest, Eötvös Sect. Math. 22-23 (1979-80), 24.3-245.

[8] Papageorgiou, N.S., Convergence theorems for Banach space valued integrable multifunctions, Intern. J. Math. and Math. Sci. 10 (1987), 433-442.

[9] Papageorgiou, N.S., Measurable multifunctions and their applications to convex integral functionals, Intern. J. Math. and Math. Sci. 12:1 (1989), 175-192.

[10] Papageorgiou, N.S., Optimal control of nonlinear evolution inclusions, J. Optim. Theory Appl. 67 (1990), 321-354.

[11] Papageorgiou, N.S., On the trajectories of controlled evolution inclusions, Comm. Math. Univ. S.P. 39 (1990), 53-67.

[12] Papageorgiou, N.S., A minimax optimal control problem for evolution inclusions, Yokohama Math. J. 39 (1991), 1-19.

[13] Papageorgiou, N.S., Continuous dependence results for a class of evolution inclusions, Proc. of the Edinburgh Math. Soc. 35 (1992), 139-158.

[14] Papageorgiou, N.S., On the set of solutions of a class of nonlinear evolution inclusions, Kodai Math. J. 15 (1992), 387-402.

[15] Simon, J., Compact sets in the space $L^{p}(0, T ; B)$, Ann. Mat. Pura Appl. 146 (1987), 65-96.

[16] Zeidler, E., Nonlinear Functional Analysis and Applications II, Springer, New York 1990. 


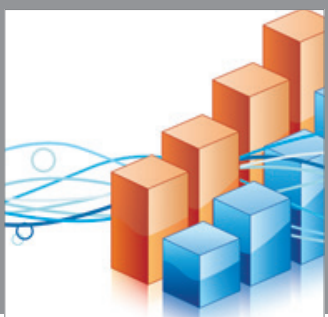

Advances in

Operations Research

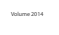

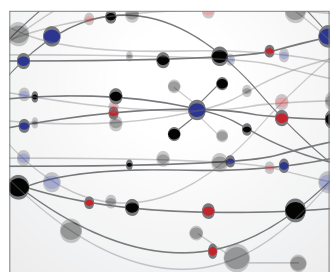

\section{The Scientific} World Journal
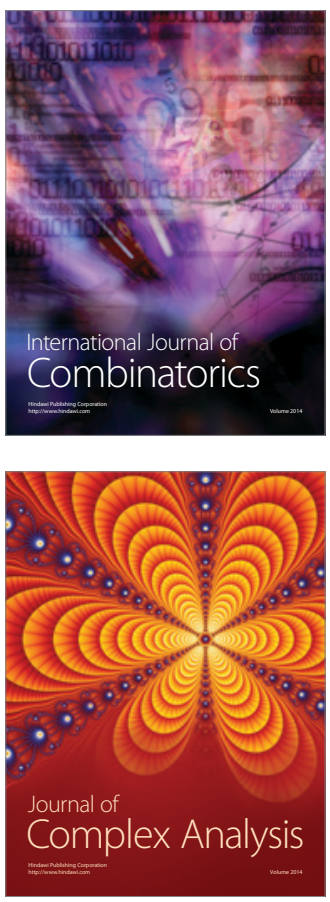

International Journal of

Mathematics and

Mathematical

Sciences
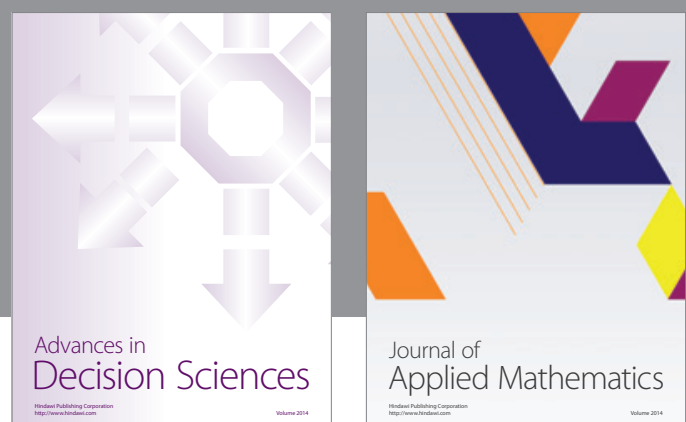

Journal of

Applied Mathematics
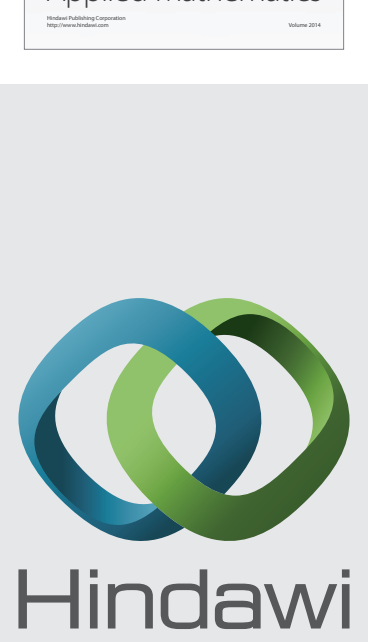

Submit your manuscripts at http://www.hindawi.com
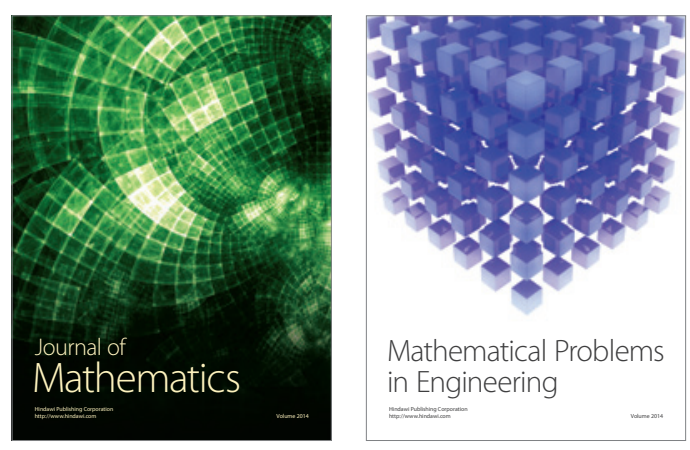

Mathematical Problems in Engineering
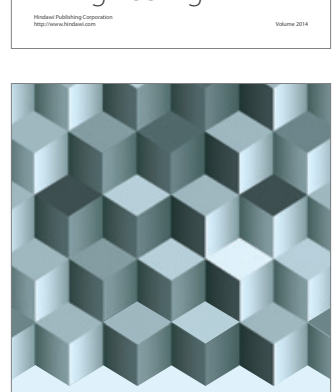

Journal of

Function Spaces
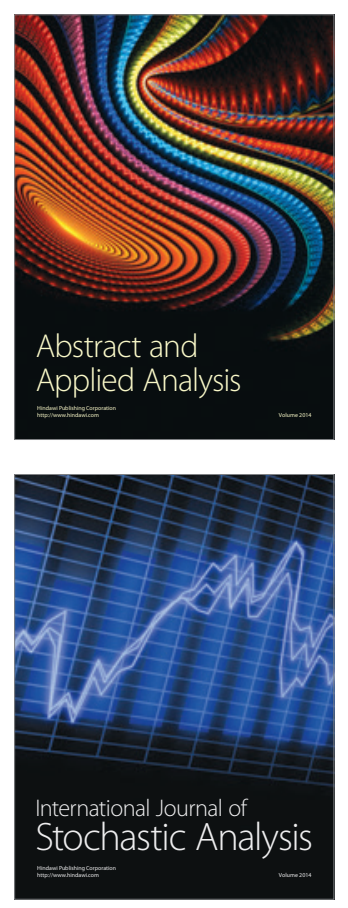

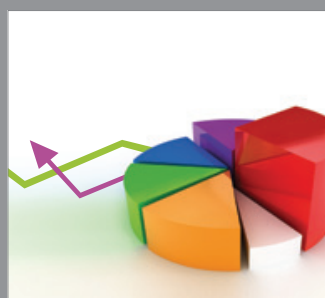

ournal of

Probability and Statistics

Promensencen
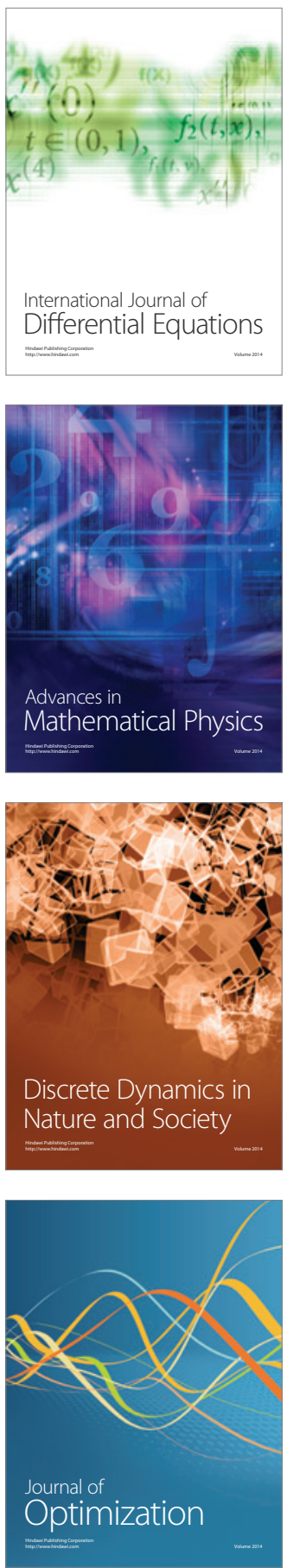